\title{
Daseinsfürsorge und Degrowth
}

\author{
Der Übergang in eine Postwachstumsgesellschaft \\ wird nur über eine große Transformation möglich \\ sein. Doch welche moralischen Forderungen \\ gehen damit einher? Und welche Auswirkungen \\ hat diese Transformation auf die soziale und \\ kulturelle Lebenswelt? \\ Von Konrad Ott und Frederike Neuber
}

\section{D} ie Degrowth (DG)-Bewegung fordert die Abkehr westlicher Gesellschaften vom Wachstumsdenken. Diese Abkehr wird häufig als "große Transformation“ bezeichnet, also als ein Gestaltwandel moderner Gesellschaften, dessen Ausmaß mit denen der Neolithisierung und der Industrialisierung vergleichbar sei. Diese Abkehr lässt sich unterschiedlich konzipieren, wobei immer moralische und politische Wertungen eingehen, die es zu explizieren und zu rechtfertigen gilt. Die Unterscheidung zwischen physischem und ökonomischem Wachstum sollte in jedem DG-Konzept Berücksichtigung finden. Ökonomisches Wachstum bezieht sich auf das BIP, physisches Wachstum auf die wirtschaftliche Inanspruchnahme natürlicher und halbkultürlicher Bestände, Fonds und Senken.

Es wird vorgeschlagen, die DG-Bewegung hinsichtlich ihrer Grundkonzepte, Framings, Ziele und Transformationspfade $\mathrm{zu}$ differenzieren (Ott 2011; 2012). Zu unterscheiden sind ein DG-Verständnis, das die Unzulänglichkeiten der Messgröße des BIP betont (DG-1), ein nachhaltigkeitstheoretisches DG-Verständnis, das einen Rückgang des physischen Ausmaßes des Wirtschaftssystems fordert (Ott/Döring 2008, DG-2), eine Konzeption, die soziokulturellen Wandel fokussiert (DG-3), und eine sozialrevolutionäre Konzeption, die in kapitalismuskritischen Traditionen verankert ist (DG-4). In jedes Konzept gehen gesellschaftstheoretische Voraussetzungen ein. Ein Vorschlag, Hegels normativ gehaltvolle Theorie moderner Gesellschaftlichkeit für eine DG-Transformation fruchtbar zu machen, findet sich in Ott (2016). Der nachfolgende Artikel wirft unter diesen Voraussetzungen und anschließend an das Projekt zu Ressourcenschonung und Postwachstum (PoWaRes) einige Schlaglichter auf sozial- und wirtschaftspolitische Probleme einer Abkehr vom ökonomischen Wachstum.

$\mathrm{Zu}$ einem DG-Konzept gehören Aussagen über die Zielfunktion, da Schrumpfung kein Selbstzweck ist. Unsere Zielfunktion lautet: Die westlichen Gesellschaften sollten ihre Wirtschaftsweisen und Lebensstile auf ein naturverträgliches Maß reduzieren und sie sollten weder in Raum noch Zeit auf Kos- ten anderer leben, das heißt weder auf Kosten zukünftiger Generationen noch auf Kosten ärmerer Gesellschaften in anderen Weltregionen. Das naturverträgliche Maß lässt sich anhand der sogenannten "planetary boundaries" und der Regeln und Leitlinien starker Nachhaltigkeit spezifizieren. Das Verbot, auf Kosten anderer zu leben, näher zu bestimmen, führt in Fragen einer politischen Ökonomie der Globalisierung.

Die konstitutive Sinndimension von DG lautet Living within limits. Diese Sinndimension impliziert Umgangsweisen mit Endlichkeit, Mangel, Begrenztheit und Knappheit, damit auch einen Sinn für Trade-Offs und Opportunitätskosten. In einer DG-Gesellschaft wäre ein verschwenderischer Umgang mit knapper werdenden Produktionsfaktoren folglich nicht ratsam. Insofern bliebe Effizienz wichtig. Fehlallokationen würde eine DG-Wirtschaftspolitik ebenso vermeiden wollen wie hohe Suchund Transaktionskosten sowie Ausweichverhalten in Richtung Schwarzmärkte. Wie viele Subventionen wofür kann und will sich eine DG-Gesellschaft leisten?

\section{Reproduktion der Lebenswelt}

Besagte Sinndimension muss die beiden wesentlichen Modi gesellschaftlicher Reproduktion prägen können: die materielle und die symbolische Reproduktion. Die symbolische Reproduktion der Lebenswelt erfolgt in den Dimensionen von kulturellen Traditionen, normativen Institutionen und erzieherischen Praktiken, während die materielle Reproduktion die Gesellschaftsmitglieder mit Gütern, Dienstleistungen und sozialen Sicherungssystemen versorgt, deren Werte erarbeitet und deren Verteilungen für gerecht erachtet werden müssen. Soziale Nachhaltigkeit bedeutet daher über die persönliche Tugend der Suffizienz hinaus die Kontinuität beider krisenanfälligen Reproduktionsweisen. Eine Gesellschaft, die sich auf das Projekt einlässt, sich selbst als Postwachstumsgesellschaft einzurichten, muss Wandel und Bestand miteinander vermitteln können. „Großer“ Wandel bedarf einer gewissen Beständigkeit des alltäglichen Lebens. Der Wandel darf nicht „aus den Fugen geraten“. Daher bedarf es verlässlicher institutionellen Fugungen. Wir denken hier aufseiten des Staates an Prinzipien des Rechtsstaates, grundgesetzliche Garantien, checks and balances, verlässliche Administration und aufseiten der Bürgerschaft an qualifizierten Rechtsgehorsam, gewaltfreie Konfliktaustragungen, subsidiarische Solidaritäten, Kulturen der Kompromissbildung, kritische Öffentlichkeit. In Abwandlung von Böckenfördes Diktum: Ein DG-Wandel lebt von kulturellen und normativen Voraussetzungen, die weder eine physische noch 
eine ökonomische Schrumpfung erzeugen können. Diese Voraussetzungen müssen während des Wandels immer wieder reproduziert werden. DG muss für die Mehrheit der Bevölkerung ein zustimmungswürdiges Projekt sein. Eine entsprechende Strategie besteht darin, die Wachstumstreiber allmählich zu entschärfen und in den Sphären von Recht, Kultur, Privatleben, Wirtschaft, Zivilgesellschaft und Politik die vielfältigen Möglichkeiten für aufeinander abgestimmte Reformen ergreifen.

$\mathrm{Zu} \mathrm{DG-Konzepten} \mathrm{gehören} \mathrm{anamnetische} \mathrm{Rückblicke} \mathrm{auf}$ die Zeiten hoher physischer und ökonomischer Wachstumsraten. Jede DG-Konzeption muss nach den Errungenschaften der Wachstumsepoche fragen und Aussagen darüber treffen, ob und inwieweit diese Errungenschaften bewahrt beziehungsweise „aufgehoben“ werden können. Zu diesen Errungenschaften zählen nicht nur Konsumstandards, sondern der Ausbau von Sozialversicherungssystemen (Renten, Gesundheit, Pflege), Wohlfahrtsstaat, kulturellen Angeboten, eines (von Kindertagesstätten bis zur Universitätslandschaft) ausgebauten Bildungssystems und die Reallohnentwicklung. Das linkspopuläre Narrativ eines Niedergangs der Institutionen der Daseinsfürsorge (IDF) halten wir mit Cremer (ebd. 2018) für unhaltbar. Ebenso falsch ist das Narrativ einer stagnierenden Reallohnentwicklung seit 1990 (Görgens 2018). Wir behaupten, dass der Ausbau der IDF in der Nachkriegszeit zu Kohäsion und Resilienz des bestehenden Gesellschaftsgefüges maßgeblich beigetragen haben. IDF sind Versicherungssysteme gegen die Risiken der Globalisierung. Sie stellen den Kern der politischen Output-Legitimation der Demokratie dar. Daher ist die Frage nach der Zukunft der IDF für jede DG-Konzeption zentral. Wir rechnen das System der IDF zu den makroökonomischen Vermittlungen zwischen Wirtschaft und Staat.

Wie also kann eine DG-Gesellschaft ein Sozialbudget in der heutigen Größenordnung gewährleisten oder aber glaubwürdige Aussichten auf funktional äquivalente Leistungen in informellen Sektoren bieten? Freilich könnte man IDF auch „mitschrumpfen“. Wären DG-Konzepte bereit, ernsthafte Einschnitte bei den heutigen IDF ins Auge zu fassen? Wäre es beispielsweise unvermeidlich, bei ökonomischer Schrumpfung in einem DG-Gesundheitswesen offener als bisher über Rationierung medizinischer Dienstleistungen bei hochbetagten Personen zu diskutieren? Wenn die heutige Gesellschaft circa $11 \%$ des BIP für Gesundheitsleistungen aufwendet und das BIP (pro Jahr oder insgesamt) um x\% schrumpfte, kann jede/r die Konsequenzen leicht berechnen. Würden in einer DG-Gesellschaft zivilisatorische Gesundheitsrisiken zurückgehen, aber gerade dadurch die Pflegekosten hochbetagter Menschen stark ansteigen? Oder wandeln sich während der großen Transformation die Vorstellungen von Gesundheit, Altern, Gebrechen, Hinfälligkeit und so weiter so grundlegend, dass eine DG-Gesellschaft auch mit, sagen wir, $10 \%$ eines geschrumpften BIP für Gesundheit „hinkäme“?

Ein DG-Staat, der hohe IDF-Standards beibehält, lässt sich nicht dauerhaft durch Substanzbesteuerung (Erbschaft, Vermögen) finanzieren. Dies schließt solche Steuern keineswegs aus, sondern nur den Glauben, sie könnten den Finanzierungsbedarf einer DG-Gesellschaft maßgeblich decken. Die Erhöhung der Erbschaftssteuer wirkt (unter realistischen Verhaltensannahmen) wie ein Konjunkturprogramm. Substanzsteuern führen womöglich also eher zu einer kurzlebigen Ökonomie der Verschwendung (im Sinne Batailles) als zu dauerhaft hohen Staatseinnahmen. Die Besteuerung von Betriebsvermögen wäre freilich ein probates Mittel, ökonomisch einen Schrumpfungskurs einzuleiten. Aber alsbald gingen dann die Steuereinnahmen an anderer Stelle drastisch zurück. Und Vermögen, die durch Besteuerung aufgezehrt worden sind, kommen nie mehr zurück. Was also soll dann besteuert werden? Die Überlegungen zu einem dauerhaft tragfähigen DG-Steuersystem stehen noch am Anfang; wichtige Impulse hierzu verdanken wir PoWaRes.

\section{Finanzierungsbedarf und Besteuerung}

Ein DG-Konzept kann wirtschaftspolitisch stärker etatistisch, kommunitaristisch oder liberalistisch sein. Gegen die Ausweitung genossenschaftlichen Wirtschaftens spricht nichts; reale commons sind allerdings strikt reguliert und solidarisch nur im Binnenbereich. Sie können Märkte nicht substituieren. Ein marktliberales DG-Konzept könnte Schumpeters Formel der „schöpferischen Zerstörung“ ummünzen. Es ginge dann um eine DG-Gründerzeit mitsamt einem Typus von Unternehmer/innen, deren Ideal nicht Gewinnmaximierung wäre, mitsamt eines „grünen“ Ordoliberalismus, der auf demokratisch legitime Weise reguliert und stimuliert. Physisches DG kann mit innovativen Formen von Bioökonomie (market gardening, Aquakultur, Recycling, vegane Gastronomie), mit cap a trade-Instrumenten und mit ökonomischer In-Wert-Setzung von sogenannten ecosystem services einhergehen. Eine DG-Gesellschaft muss politisch entscheiden, ob und inwieweit diesbezügliche Geschäftsideen zulässig und wünschenswert sind.

DG umfasst die Transformation der kulturellen und sozialen Lebenswelt. Suffizient lebende Bürger würden nur kaufen, was sie wirklich bräuchten, und würden vielerlei selber produzieren oder reparieren. Sie würden sparsam mit Ressourcen umgehen - also im besten Sinne „ökonomisch“ handeln. Die von Aristoteles stammende Unterscheidung von Hausund Geldwirtschaft würde sich in einer DG-Konsumkultur auf neue Weise vermitteln. Würde sich hierdurch der Trend zur Dienstleistungsgesellschaft fortsetzen? Hier melden sich second thoughts. Welche Dienstleistungen würde ein/e Bürger/ in in einer DG-Kultur in Anspruch nehmen? Würde er/sie ihre Kinder in die Ganztagesbetreuung geben, wenn sie nur fünf Stunden am Tag arbeiten würde? Welche Dienstleistungen also würden wirklich nachgefragt und wie entwickelten sich die Preise für Dienstleistungen an Märkten? Vielleicht wäre es bei einem Überangebot an Dienstleistungen lukrativer, Schuster/in oder Schneider/in zu werden.

Große Wandlungen erhöhen, geschichtlich gesehen, die Ungleichheit unter den Menschen. So haben sowohl das Neo- 
lithikum als auch die Industrialisierung die Ungleichheit zunächst erhöht. Dies gilt auch für die postsowjetische und postmaoistische Gesellschaft. Der Übergang in eine DG-Gesellschaft soll nun aber in vielen DG-Konzepten, vielleicht mit Ausnahme des Konzepts von Meinhard Miegel, mit einer deutlichen Verringerung der heutigen Ungleichheit einhergehen.

\section{Wandlungen und Ungleichheit}

Problematisch dabei ist, dass eine DG-Gesellschaft, die ökonomisch schrumpft, ceteris paribus die bestehenden Vermögen relativ erhöht, da die Diskontrate negativ wird. Dieser Effekt läuft einer egalitären DG-Moral zuwider. Folglich muss die Umverteilung der Vermögen intensiviert werden im Wissen darum, dass sich Verteilungskonflikte bei wachsender Knappheit tendenziell verschärfen. Was verursacht im Übergang zu einer DG-Gesellschaft härtere Konflikte: Ungleichheit oder deren Verringerung durch Umverteilung? Wäre es denkbar, dass viele Personen einer DG-Gesellschaft wenig Wert darauf legten, ihr Güterbündel mit Güterbündeln anderer Personen zu vergleichen? Genügsame, zufriedene Menschen könnten durchaus Anti-Egalitarist/innen (im Sinne von Harry Frankfurt) sein.

Eine DG-Konzeption muss nicht zuletzt auf die moralischen Forderungen reflektieren, die sie erhebt. Was wäre, wenn es zuträfe, dass altruistisches und solidarisches Handeln in Low-Cost-Situationen wahrscheinlicher wird, die Kosten für solches Handeln jedoch in einer DG-Gesellschaft steigen? Francis Bacon glaubte, technischer und wirtschaftlicher Fortschritt würde die Großherzigkeit der Menschen befördern, da Wohlstand das Teilen und Schenken leichter macht. Wie kann man verhindern, dass sich Einstellungen wie Geiz und Hartherzigkeit verbreiten? Was werden wir in einer DG-Gesellschaft einander schenken? Würde es in einer ökonomisch schrumpfenden DG-Gesellschaft viele Umsonst-Läden geben - oder keine?
Die Liste der Fragen ließe sich verlängern: Zuwanderung, Kriminalitätsraten, Landnutzung, Naturschutz, Medien, Pädagogik, auswärtige Beziehungen und vieles mehr. Eine DG-Gesellschaft wird kein Schlaraffenland sein. Grundfalsch ist es demnach, Denkfiguren der Überflussgesellschaft in DG-Konzepte hineinzuprojizieren. Deshalb sind alle Konzepte fragwürdig, die die Bilderwelten der utopischen Tradition, wie sie Ernst Bloch im „Prinzip Hoffnung“ ausgebreitet hat, in den Fokus eines DG-Imaginären rücken.

\section{Literatur}

Cremer, G. (2018): Deutschland ist gerechter, als wir meinen. München, Beck. Görgens, H. (2018): Irrtum und Wahrheit über die Reallohnentwicklung seit 1990. Marburg, Metropolis.

Ott, K./Döring, R. (2008): Theorie und Praxis starker Nachhaltigkeit. Marburg, Metropolis.

Ott, K. (2012): Variants of degrowth and deliberative democracy. A Habermasian proposal. In: Futures 44/6: 571-581.

Ott, K. (2011): Vier Pfade ins Postwachstumszeitalter. In: Vorgänge 195: 54-69.

Ott, K. (2016): Sittlichkeit und Nachhaltigkeit in einer Postwachstumsgesellschaft. Eine Forschungsagenda. In: Bihrer, A./Franke-Schwenk, A./Stein, T. (Hrsg.): Endlichkeit. Bielefeld, Transcript. 150-178.

\section{AUTOR/INNEN + KONTAKT}

Dr. Konrad Ott ist Professor am Lehrstuhl Philosophie und Ethik der Umwelt an der Christian-Albrechts-Universität zu Kiel.

Christian-Albrechts-Universität zu Kiel, Christian-Albrechts-Platz 4, 24118 Kiel.

Tel.: +49 431 8802822, E-Mail: ott@philsem.uni-kiel.de, Website: www.uni-kiel.de/de

Dr. Frederike Neuber ist freie Wissenschaftlerin und Workshopleiterin zu den Themen Philosophie,

Rationales Argumentieren und Klimaschutz.
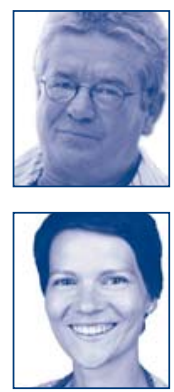

Frederike Neuber, Österfeldstraße 15b, 70563 Stuttgart. Tel.: +49 711 88882814, E-Mail: frederike.neuber@gmx.net, Website: www.frederikeneuber.de
Nachhaltigkeit

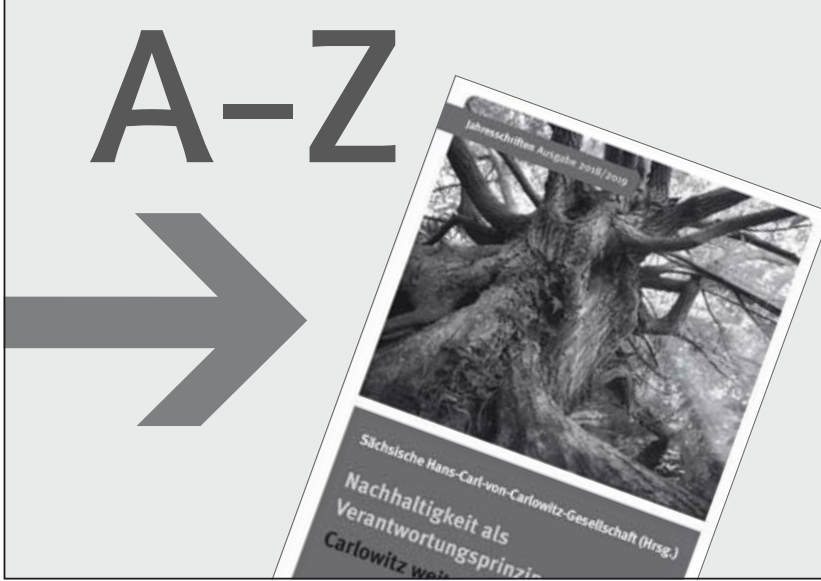

\section{H wie Horizonte}

Nachhaltige Strukturen zu etablieren, zu hüten, zu pflegen und wachsen zu lassen kann verhindern, dass die Welt weiter aus den Fugen gerät - auf allen Ebenen, lokal wie global. Diese Gewissheit haben die Autorinnen und Autoren der diesjährigen Jahresschrift gemeinsam. Sie arbeiten auf unterschiedlichen Wegen an verschiedenen Brennpunkten unseres Planeten beispielhaft an der Lösung gemeinsamer Probleme.

Sächsische Hans-Carl-von-Carlowitz-Gesellschaft (Hrsg.)

Nachhaltigkeit als Verantwortungsprinzip

Carlowitz weiterdenken

204 Seiten, broschiert, 15,- Euro, ISBN 978-3-96238-029-8

Erhältlich im Buchhandel oder versandkostenfrei

innerhalb Deutschlands bestellbar unter www.oekom.de

Die guten Seiten der Zukunft 